\title{
Qualidade de vida de idosos atendidos em Programa de Assistência Domiciliária
}

Quality of life of elderly patients assisted by a Home Care Program

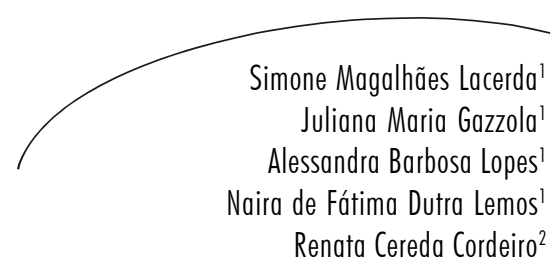

Resumo

Objetivo: Avaliar a qualidade de vida de idosos em assistência domiciliária. Método: estudo transversal analítico. Foram avaliados idosos de um programa de assistência domiciliária quanto aos dados sociodemográficos, clínicos, funcionais e de qualidade devida pelo instrumento WHOQOL-Bref. Foram excluídos idosos com déficit cognitivo e/ou déficit auditivo grave. Utilizou-se o teste de Mann Whitney, Kruskal-Wallis e correlação de Spearman para verificar a associação do $W H O Q O L-B r e f$ com as variáveis $(\alpha=0,05)$. Resultado: a amostra foi constituída por 23 idosos, com média etária de $84,22 \pm 7,89$ anos, maioria feminina $(69,6 \%)$. O número de comorbidades encontrado foi de $7,13 \pm 2,32$. O tempo médio de dependência funcional foi de $3,48 \pm 2,5$ anos. As variáveis que se aproximaram dos domínios do WHOQOL-Bref foram: sintomas depressivos ( $\mathrm{p}<0,001)$, número de comorbidades $(\mathrm{p}=0,037)$ e capacidade funcional $(\mathrm{p}=0,019)$ com o domínio físico; idade $(\mathrm{p}=0,015)$, capacidade funcional $(\mathrm{p}=0,004)$, número de medicamentos $(p=0,037)$ e sintomas depressivos $(p=0,002)$ com o psicológico; idade $(\mathrm{p}=0,002)$, sintomas depressivos $(\mathrm{p}=0,002)$, número de comorbidades $(\mathrm{p}=0,010)$ com o meio-ambiente; afecções psiquiátricas $(\mathrm{p}=0,016)$ e capacidade funcional $(\mathrm{p}=0,001)$ com o domínio relações sociais; e queixa de dor $(\mathrm{p}=0,002)$, escala visual analógica de dor $(\mathrm{p}=0,021)$, número de medicamentos $(\mathrm{p}=0,002) \mathrm{e}$ sintomas depressivos $(\mathrm{p}=0,050)$ com o domínio geral (overall). Conclusão: os fatores que influenciam negativamente a $\mathrm{QV}$ de idosos em $\mathrm{AD}$ são: idosos mais jovens, queixa de dor em maior intensidade, polifarmacoterapia, maior número comorbidades, presença de sintomas depressivos e outras afecções psiquiátricas e comprometimento funcional.

\footnotetext{
Universidade Federal de São Paulo. Escola Paulista de Medicina, Disciplina de Geriatria e Gerontologia. São Paulo, SP, Brasil.

2 Universidade Federal de São Paulo. Escola Paulista de Medicina, Departamento de Medicina Preventiva. Setor de Reabilitação Gerontológica - Lar Escola São Francisco. São Paulo, SP, Brasil.
}

\section{Palavras-chave:}

Cuidadores.

Envelhecimento. Amostra. Serviços de saúde para idosos. Idoso fragilizado. Pacientes domiciliares. 


\section{Abstract}

Objective: To evaluate the quality of life of elderly patients in home care. Method: Analytical cross-sectional study. Elderly patients of a home care program were assessed regarding socio-demographic, clinical, functional data and quality of life by the WHOQOL-Bref. Elderly people with cognitive and/or severe hearing deficits were excluded from the study. The T-Student, ANOVA and Pearson's Correlation tests were used to investigate the association between the WHOQOLBref and the variables $(\alpha=0.05)$. Results: The sample comprised 23 elderly patients, mostly female $(69.6 \%)$, with mean age of $84.22 \pm 7.89$ years. The number of comorbidities found was $7.13 \pm 2.32$. The mean time of functional dependence was $3.48 \pm 2.5$ years. The variables that presented significant association with the WHOQOL-Bref domains were: depressive symptoms $(\mathrm{p}<0.001)$, number of illnesses $(p=0.037)$ and functional capacity $(p=0.019)$ with the physical domain; age $(p=0.015)$, functional capacity $(p=0.004)$, number of drugs $(p=0.037)$ and depressive symptoms $(p=0.002)$ with the psychological domain; age $(p=0.002)$, depressive symptoms $(p=0.002)$, number of illnesses $(p=0.010)$ with the environment domain; psychiatric affections $(\mathrm{p}=0.016)$ and functional capacity $(p=0.001)$ with the social relationships domain; and pain complaint $(p=0.002)$, analogical visual scale of pain $(p=0.021)$, number of drugs $(p=0.002)$ and depressive symptoms $(\mathrm{p}=0.050)$ with the overall domain ("overall”). Conclusion: Factors that negatively influence the QoL of elderly patients in $\mathrm{HC}$ are: young elderly, high intensity pain complaint, poly-pharmacotherapy, high number of illnesses, presence of depressive symptoms and other psychiatric affections, and functional deficit.
Key words: Caregivers. Aging. Sample Size. Health of the elderly. Frail elderly. Homebound persons.

\section{INTRODUÇÃO}

O envelhecimento populacional proporciona um aumento da prevalência de doenças crônicas e incapacitantes, resultando em mudança no paradigma da saúde pública. A saúde não é mais medida pela presença ou não de doenças, e sim pelo grau de preservação da capacidade funcional. ${ }^{1}$

Dependendo do grau de incapacidade, o idoso pode perder a independência (capacidade de recorrer aos seus próprios meios) e autonomia (a faculdade de se governar por si mesmo), ${ }^{2}$ requerendo cuidados especiais que podem ser contínuos, prolongados e de alto custo. Desta forma, a assistência domiciliária (AD) é uma alternativa promissora, pois é menos onerosa ao sistema de saúde e de qualidade, podendo minimizar as perdas dos idosos. ${ }^{3}$

A AD insere-se dentro de um modelo gerontológico que visa, na medida do possível, a reintegrar o idoso na comunidade, preservando ao máximo sua autonomia, buscando a recuperação de sua independência funcional ${ }^{4}$ e procurando mantê-lo um "cidadão ativo, participativo, produtivo e afetivo". ${ }^{5}$ Ao se discutir o conceito de $\mathrm{AD}$, deve-se levar em consideração o conteúdo da assistência, as medidas de resultado e os objetivos subjacentes. Thome et al., ${ }^{6}$ ao revisarem sistematicamente a literatura acerca do tema $\mathrm{AD}$, notaram que, em relação ao conteúdo da assistência, as ações levam em consideração as necessidades individuais que variam desde a prevenção primária até os cuidados paliativos na terminalidade da vida. Em relação aos resultados, essa assistência tem por metas produzir impacto sobre o indivíduo e sobre a saúde pública. Indiretamente, seus benefícios individuais e coletivos coincidem, pois partem do princípio que no ambiente domiciliar tanto a capacidade funcional, como a qualidade de vida, aumentam ou podem ser mantidas, minimizando a necessidade de reinternações 
hospitalares, reduzindo sua permanência e possivelmente seus custos implicados. ${ }^{6}$

A expressão qualidade de vida (QV) foi cunhada em 1920 por Redou em seu livro The Economics of Welfare, e, após a Segunda Guerra Mundial, com o redimensionamento do conceito de saúde pela Organização Mundial da Saúde (OMS), incluiu-se o bem-estar físico, mental e social. ${ }^{7}$ Embora não haja consenso a respeito do conceito de QV, três aspectos fundamentais são considerados: subjetividade, multidimensionalidade e presença de dimensões positivas e negativas. ${ }^{8}$

Avaliações da QV, por meio da percepção de saúde dos indivíduos, têm se tornado o foco das pesquisas sobre a efetividade de serviços de saúde. A busca de um instrumento que avaliasse a QV dentro de uma perspectiva genuinamente internacional fez com que a OMS organizasse um projeto colaborativo multicêntrico que elaborou o questionário WHOQOL-100. ${ }^{8} \mathrm{~A}$ versão abreviada, WHOQOL-Bref, consta de 26 questões, sendo duas questões gerais (geral) e 24 questões representando cada uma das 24 facetas que compõe o instrumento original, relacionadas ao domínio físico, psicológico, relações sociais e meio ambiente, mantém as características psicométricas satisfatórias em um breve tempo de aplicabilidade. ${ }^{8} \mathrm{O} \mathrm{WHOQOL}$ Bref, validado transculturalmente para sua aplicação no Brasil, e suas questões foram desenvolvidas a partir do princípio de que o conceito de QV é abrangente e pode ser aplicado a várias doenças e situações não médicas, inclusive em populações específicas, como é o caso da idosa, alvo do presente estudo. ${ }^{9} \mathrm{Na}$ população idosa, a escala mostra adequadas propriedades psicométricas, inclusive validade discriminante para os níveis mais graves de sintomas depressivos, desesperança e percepção do estado de saúde. ${ }^{9}$

Este estudo se justifica pela necessidade de avaliar a QV em idosos atendidos por um programa de $\mathrm{AD}$, com a finalidade de apontar elementos que devem ser considerados no planejamento das ações do atendimento domiciliário ao idoso, pelas equipes interdisciplinares, permitindo assim restaurar, manter e melhorar a QV dessa população. É sabido que essa modalidade de atendimento está em franco crescimento no Brasil. Estudos nacionais e internacionais que associem a ideia dos cuidados domiciliares sob os quais os idosos estão submetidos à qualidade de vida desses beneficiários, em seus aspectos mais abrangentes, não são suficientemente numerosos ou consistentes para elucidar melhor as necessidades dessa população dentro dessa modalidade de assistência. $^{6}$

Deste modo, o presente estudo tem como objetivo avaliar a QV de pacientes idosos atendidos por um programa de $\mathrm{AD}$ por meio do instrumento $\mathrm{WHOQOL-Bref}$.

\section{MATERIAL E MÉTODO}

Foi realizado estudo transversal analítico no Programa de Assistência Domiciliar ao Idoso (PADI). Este estudo foi aprovado pelo Comitê de Ética em Pesquisa da Unifesp (protocolo número 1279/05) e os participantes assinaram o termo de consentimento livre e esclarecido. O PADI foi implantado em 1999 na disciplina de Geriatria e Gerontologia da Universidade Federal de São Paulo - Unifesp, e atende pacientes cujas condições clínicas e sociais impedem o atendimento ambulatorial. São critérios de inclusão: residir num raio de até oito quilômetros da Unifesp, ter um cuidador e necessitar de cuidados que possam ser prestados com segurança no domicílio. O critério do perímetro foi estabelecido a partir da necessidade de delimitação de uma área para atendimento à população, que permitisse a realização de um número adequado de visitas por período, considerando o trajeto e o trânsito no município de São Paulo. Seus objetivos são: promover a individualização e humanização do atendimento, respeitar a memória física e afetiva da pessoa idosa, reduzir o número de internações, reforçar vínculos familiares e sociais e orientar cuidadores e familiares.

Os critérios para inclusão na presente pesquisa consideraram idosos com idade igual ou superior 
a 60 anos, dos gêneros masculino e feminino e em atendimento pelo PADI. Foram excluídos os idosos com impossibilidade de responder aos questionários propostos para a avaliação em função de déficit auditivo grave e/ou demência moderada a grave. Foram selecionados para a entrevista somente os idosos com pontuação acima de 13 pontos no Mini-Exame do Estado Mental (MEEM), ${ }^{10}$ de acordo com os dados recolhidos previamente no prontuário médicos dos pacientes. No estudo de Bertolucci et al., ${ }^{10}$ indivíduos normais foram comparados a indivíduos com diferentes causas de comprometimento cognitivo, obtendo-se boas sensibilidades e especificidades para os níveis de corte; porém, para o grupo analfabeto, este se revelou extremamente baixo (escore de corte de 13 pontos).

Grande parte dos idosos atendidos em AD apresenta algum grau de comprometimento cognitivo. Deste modo, avaliar subjetivamente a QV de idosos com demência leve a moderada é o melhor meio de compreender sua experiência de vida, e o uso de ferramentas subjetivas como questionários multidimensionais é justificável..$^{11} \mathrm{No}$ estudo de Longsdon et al., ${ }^{12}$ apenas os idosos cujos escores no MEEM estiveram abaixo de 10 não conseguiram completar o questionário proposto.

A depressão e a demência podem distorcer a percepção de qualidade de vida, contudo, não invalidam sua avaliação sob o olhar do próprio sujeito. ${ }^{13}$ Foi realizado roteiro estruturado para coleta dos dados sociodemográficos e clínicos dos idosos. Os dados sociodemográficos analisados foram gênero, idade em anos completos, escolaridade em anos de estudo, arranjo (família multigeracional, cônjuge ou outros de sua geração e tipo de moradia, estado civil, situação socioeconômica (renda familiar), atividades de lazer, religião e prática religiosa. Os dados clínicos analisados foram: número de comorbidades, quantidade de medicamentos e internações hospitalares nos últimos seis meses, presença de afecções musculoesqueléticas, cardiovasculares, pulmonares, psiquiátricas (incluindo depressão), renais e endocrinometabólicas, percepção subjetiva da visão e da audição, queixa e tempo de dor, uso de dispositivo de auxílio à marcha, tempo de dependência funcional, escala visual analógica $(\mathrm{EVA})^{14}$ de dor, escala de depressão geriátrica e capacidade funcional.

Os instrumentos utilizados neste estudo para avaliação da capacidade funcional foi o Brazilian OARS Multidimensional Functional Assesment Questionnaire-BOMFAQ; ${ }^{15}$ para avaliação subjetiva da depressão, foi utilizada a Escala de Depressão Geriátrica Abreviada. ${ }^{16}$

O BOMFAQ ${ }^{15}$ avalia a dificuldade referida na realização de 15 atividades de vida e sete atividades instrumentais de vida diária (AIVD). Foi quantificado o total de AVDs que o paciente referiu dificuldade para realizá-las, ou seja, o total de atividades comprometidas. Quanto maior a pontuação, maior o comprometimento $\mathrm{da}$ capacidade funcional.

A Escala de Depressão Geriátrica Abreviada (GDS-15) é um questionário de 15 perguntas com respostas dicotômicas (sim ou não) a respeito de como a pessoa idosa tem se sentido durante a última semana. Trata-se de uma ferramenta útil de rastreamento para facilitar a avaliação de sinais de depressão em idosos. Em relação à pontuação, de 0 a 4 pontos é considerado não deprimidos; de 5 a 10, indícios de depressão leve ou moderada; e de 11 a 15 indícios de depressão grave ou severa. ${ }^{16}$

Para avaliar a QV, foi utilizado o instrumento WHOQOL-Bref, ${ }^{8}$ cuja versão em português foi validada em 1998. ${ }^{17}$ Trata-se de 26 questões, das quais as duas primeiras se referem ao domínio geral da QV ("como você avaliaria sua qualidade de vida?" e "quão satisfeito você está com sua saúde?”). Os demais domínios são: físico, psicológico, relações sociais e meio ambiente, relacionados às 24 questões restantes. Nesse instrumento, os escores finais variam de 4 a 20 pontos comparáveis ao WHOQOL-100, transformados em escala de $\mathrm{O}$ a 100 (quanto maior o escore melhor a QV). Não existem pontos de corte que determinem um escore abaixo ou acima do qual se possa avaliar a QV como "ruim" ou "boa", dependendo exclusivamente do contexto de que se trata a avaliação, seja na comparação entre grupos de indivíduos ou prospectivamente. ${ }^{8}$ 
A análise estatística foi feita por meio de estatística descritiva simples para caracterização $\mathrm{da}$ amostra. As variáveis referentes aos domínios (geral, físico, psicológico, relação social e meio ambiente) do instrumento $\mathrm{WHOQOL-Bref}$ foram comparadas com as demais variáveis do estudo para avaliar os fatores de impacto na QV em idosos atendidos em AD. Os testes utilizados foram Correlação de Spearman, Mann-Whitney e Kruskal-Wallis, com nível de significância fixado em 0,05.

\section{RESULTADOS}

Foram avaliados 23 pacientes atendidos pelo PADI, sendo que a média etária foi de $84,22 \pm$ 7,89 anos, variando entre 62 e 99 anos e $69,6 \%$ são do gênero feminino e $82,6 \%$ brancos. O número médio de anos de estudo foi de 4,38 \pm 2,91 anos. O tempo mínimo de estudo formal variou de um a 15 anos. A renda familiar média foi de 3,41 salários mínimos e as demais variáveis sociodemográficas estão apresentadas na tabela 1 .

Tabela 1 - Variáveis sociodemográficas de idosos atendidos em serviço de assistência domiciliária $(\mathrm{n}=23)$. São Paulo, SP.

\begin{tabular}{llcc}
\hline & \multicolumn{1}{c}{ Categorias } & $\mathrm{N}$ & $\%$ \\
\hline \multirow{2}{*}{ Gênero } & Masculino & 7 & 30,4 \\
& Feminino & 16 & 69,6 \\
& & & \\
Estado Civil & Com Companheiro & 6 & 26,1 \\
& Sem Companheiro & 17 & 73,9 \\
& & & \\
Arranjo de Moradia & Cônjuge & 6 & 26,1 \\
& Multigeracional & 17 & 73,9 \\
& & & \\
Tipo de Moradia & Casa & 11 & 47,8 \\
& Apartamento & 7 & 30,4 \\
& Sobrado & 5 & 21,7 \\
\cline { 2 - 4 } & Total & 23 & 100,0 \\
\hline
\end{tabular}

A média do número de comorbidades foi de 7,13 $\pm 2,32$. As afecções apresentadas pelos pacientes estão listadas na tabela 2, sendo que as afecções musculoesqueleticas são as mais comuns, sendo referidas por $95,7 \%$ dos participantes. A percepção subjetiva da visão foi referida como "excelente ou boa" por 15 idosos $(65,2 \%)$ e como "mais ou menos, ruim ou péssima” por oito idosos $(34,5 \%)$. A percepção subjetiva audição foi referida como "excelente ou boa" por 17 idosos $(73,9 \%)$ e como "mais ou menos, ruim ou péssima” por seis idosos (26,1\%). As afecções psiquiátricas estão presentes em $8,7 \%$ dos idosos. 
Tabela 2 - Afecções clínicas de idosos atendidos em serviço de assistência domiciliária ( $n=23)$. São Paulo, SP.

\begin{tabular}{|c|c|c|c|}
\hline Afecções & Categorias & $\mathrm{N}$ & $\%$ \\
\hline \multirow[t]{2}{*}{ Afecções Musculoesqueléticas } & Presente & 22 & 95,7 \\
\hline & Ausente & 1 & 4,3 \\
\hline \multirow[t]{2}{*}{ Afecções Cardiovasculares } & Presente & 19 & 82,6 \\
\hline & Ausente & 4 & 17,4 \\
\hline \multirow[t]{2}{*}{ Afecções Pulmonares } & Presente & 3 & 13,0 \\
\hline & Ausente & 20 & 87,0 \\
\hline \multirow[t]{2}{*}{ Afecções Psiquiátricas } & Presente & 14 & 60,9 \\
\hline & Ausente & 9 & 39,1 \\
\hline \multirow[t]{2}{*}{ Demência leve } & Presente & 10 & 43,5 \\
\hline & Ausente & 13 & 56,5 \\
\hline \multirow[t]{2}{*}{ Depressão } & Presente & 13 & 56,5 \\
\hline & Ausente & 10 & 43,5 \\
\hline \multirow[t]{2}{*}{ Afecções Renais } & Presente & 2 & 8,7 \\
\hline & Ausente & 21 & 91,3 \\
\hline \multirow[t]{3}{*}{ Afecções Endocrinometabólicas } & Presente & 11 & 47,8 \\
\hline & Ausente & 12 & 52,2 \\
\hline & Total & 23 & 100,0 \\
\hline
\end{tabular}

O número médio de medicamentos utilizados por paciente foi de $5,68 \pm 2,20$, sendo que o número mínimo de medicamentos foi de 0 e o máximo de 9; e as internações hospitalares nos últimos seis meses ocorreram em 17,4\% dos 23 idosos. A queixa de dor foi referida por $65,2 \%$ dos 23 pacientes, sendo a dor em coluna lombar e MMII as mais comumente citadas e a média do tempo de dor foi de 7,36 \pm 9,89 anos. O tempo médio de dependência funcional foi de 3,48 $\pm 2,50$ anos, sendo que $73,9 \%$ da amostra de 23 pacientes avaliados faziam uso de dispositivo de auxílio à marcha. Em relação à capacidade funcional, avaliada por meio do $B O M F A Q$, os idosos referiram dificuldade em média de 11,61 atividades e DP de 2,23, com variação de seis a 15 atividades comprometidas. $\mathrm{Na}$ avaliação para rastreio de depressão em idosos, os idosos apresentaram em média de 5,22 pontos e DP de 2,81, com variação de 1 a 10 . Onze casos $(47,8 \%)$ pontuaram ente $\mathrm{O}$ a 4 (sem sinais depressivos) e 12 casos (52,2\%) pontuaram entre 6 a 10 (depressão leve ou moderada).

Quanto às atividades de lazer, dos 23 participantes da pesquisa, $87,0 \%$ dos idosos referiram ouvir rádio ou assistir televisão, 8,7\% realizam trabalhos domésticos (manuais) e 4,3\% fazem meditação. Em relação à religião, 52,2\% tinham prática religiosa e destes $91,3 \%$ eram católicos.

Todos os pacientes referiram estar satisfeitos com os cuidados recebidos em casa e com o cuidador, sendo importante ressaltar que $87,0 \%$ dos cuidadores são informais. 
A QV geral dos idosos que referiram queixa de dor apresentaram pior QV quando comparados aos que não a referiram. (tabela 3) Quanto maior a pontuação na EVA de dor, pior a QV. Quanto maior o número de medicamentos utilizados, pior a QV. Quanto maior a pontuação na Escala de Depressão Geriátrica (EDG), pior a QV. (Tabela 4)

Tabela 3 - Médias, Desvio-padrão e análises comparativas significantes dos resultados do WHOQOLBrefe as variáveis categóricas de idosos atendidos em serviço de assistência domiciliária $(n=23)$. São Paulo, SP.

\begin{tabular}{|c|c|c|c|c|c|}
\hline WHOQOL-Bref & Variáveis & Categorias & $\begin{array}{l}\text { Média (Desvio } \\
\text { Padrão) }\end{array}$ & Mediana & $\begin{array}{c}\text { Teste Mann- } \\
\text { Whitney } \\
\text { (p-valor) }\end{array}$ \\
\hline \multirow{2}{*}{ Geral } & \multirow{2}{*}{ Queixa de dor } & Presente & $55,83(12,38)$ & 50,00 & \multirow{2}{*}{$p=0,002$} \\
\hline & & Ausente & $73,43(8,01)$ & 75,00 & \\
\hline \multirow{2}{*}{ Físico } & \multirow{2}{*}{$\begin{array}{c}\text { Escala de Depressão } \\
\text { Geriátrica }\end{array}$} & 0 a 4 pontos & $58,44(7,70)$ & 57,14 & \multirow{2}{*}{$p=0,003$} \\
\hline & & 5 a 10 pontos & $47,02(6,78)$ & 48,21 & \\
\hline \multirow{2}{*}{ Psicológico } & \multirow{2}{*}{$\begin{array}{l}\text { Escala de Depressão } \\
\text { Geriátrica }\end{array}$} & 0 a 4 pontos & $57,57(12,04)$ & 58,33 & \multirow{2}{*}{$p=0,013$} \\
\hline & & 5 a 10 pontos & $45,48(10,42)$ & 47,91 & \\
\hline \multirow{2}{*}{ Relações Sociais } & \multirow{2}{*}{ Afecções Psiquiätricas } & Presente & $58,03(11,14)$ & 58,33 & \multirow{2}{*}{$p=0,016$} \\
\hline & & Ausente & $70,83(10,62)$ & 75,00 & \\
\hline
\end{tabular}

Tabela 4 - Correlação entre WHOQOL-Brefe as variáveis quantitativas de idosos atendidos em serviço de assistência domiciliária $(n=23)$. São Paulo, SP.

\begin{tabular}{|c|c|c|c|}
\hline WHOQOL-Bref & Variáveis & $\mathrm{CC}^{*}$ & p-valor \\
\hline \multirow[t]{3}{*}{ Geral } & Escala Visual Analógica de Dor & $r=-0,487$ & $\mathrm{p}=0,021$ \\
\hline & Número de medicamentos & $r=-0,626$ & $\mathrm{p}=0,002$ \\
\hline & Escala de Depressão Geriátrica & $r=-0,414$ & $\mathrm{p}=0,050$ \\
\hline \multirow[t]{3}{*}{ Físico } & Número de comorbidades & $r=0,438$ & $p=0,037$ \\
\hline & Capacidade funcional & $r=-0,485$ & $\mathrm{p}=0,019$ \\
\hline & Escala de Depressão Geriátrica & $r=-0,770$ & $\mathrm{p}<0,001$ \\
\hline \multirow[t]{4}{*}{ Psicológico } & Idade & $r=0,500$ & $\mathrm{p}=0,015$ \\
\hline & Número de medicamentos & $r=-0,446$ & $\mathrm{p}=0,037$ \\
\hline & Capacidade funcional & $r=-0,572$ & $\mathrm{p}=0,004$ \\
\hline & Escala de Depressão Geriátrica & $r=-0,610$ & $\mathrm{p}=0,002$ \\
\hline \multirow[t]{2}{*}{ Relações Sociais } & Capacidade funcional & $r=-0,624$ & $p=0,001$ \\
\hline & Idade & $r=0,659$ & $p=0,001$ \\
\hline \multirow[t]{2}{*}{ Meio-ambiente } & Número de comorbidades & $r=0,528$ & $\mathrm{p}=0,010$ \\
\hline & Escala de Depressão Geriátrica & $r=-0,608$ & $\mathrm{p}=0,002$ \\
\hline
\end{tabular}

* CC: Coeficiente de Correlação de Spearman. 
No domínio físico, quanto maior a pontuação na EDG, pior a QV. Quanto maior o comprometimento da capacidade funcional, pior a QV. Quanto maior o número de comorbidades, pior a QV. (Tabelas 3 e 4)

No domínio psicológico, os idosos que pontuaram "5 a 10 na EDG" apresentaram pior $\mathrm{QV}$ quando comparados aos que pontuaram " $\mathrm{O} a$ 4 na EDG”. (tabela 3) Quanto maior a pontuação na EDG, pior a QV. Quanto menor a idade, pior a QV. Quanto maior o número de medicamentos utilizados, pior a QV. Quanto maior o comprometimento da capacidade funcional, pior a QV. (Tabela 4)

No domínio relações sociais, os pacientes que apresentam afecção psiquiátrica têm pior a QV quando comparados aos que não apresentam. (tabela 3) Quanto maior o comprometimento da capacidade funcional, pior a QV (tabela 4).
No domínio meio ambiente, quanto menor a idade, pior a QV. Quanto menor o número de comorbidades, pior a QV. Quanto maior a pontuação na EDG, pior a QV (tabela 4).

Não foram encontradas associações significantes entre as variáveis "overall/geral" e referentes aos domínios (físico, psicológico, relações sociais e meio ambiente) do instrumento WHOQOL-Brefe as demais variáveis do estudo.

Apenas um paciente não apresentou afecções musculoesqueléticas. Os pacientes com presença destas afecções tiveram as seguintes pontuações: overall/geral $(61,36 \pm 13,86)$, físico $(51,62 \pm 8,37)$, psicológico $(50,94 \pm 12,78)$, ambiental $(55,39 \pm 10,87)$ e social $(62,12 \pm 11,91)$.

Observou-se que as médias encontradas pelos diferentes domínios foram semelhantes, o que pode sinalizar certa homogeneidade entre os aspectos analisados no presente estudo (tabela 5).

Tabela 5 - Valores descritivos dos diferentes domínios do WHOQOL-Bref de idosos atendidos em serviço de assistência domiciliária $(\mathrm{n}=23)$. São Paulo, SP

\begin{tabular}{|c|c|c|}
\hline Domínios do WHOQOL-Bref & & \\
\hline \multirow[t]{5}{*}{ Físico } & Média & 52,48 \\
\hline & Mediana & 50,00 \\
\hline & Desvio-padrão & 9,16 \\
\hline & Mínimo & 35,71 \\
\hline & Máximo & 71,43 \\
\hline \multirow[t]{5}{*}{ Psicológico } & Média & 51,26 \\
\hline & Mediana & 50,00 \\
\hline & Desvio-padrão & 12,59 \\
\hline & Mínimo & 29,17 \\
\hline & Máximo & 83,33 \\
\hline \multirow[t]{5}{*}{ Relações Sociais } & Média & 63,04 \\
\hline & Mediana & 66,66 \\
\hline & Desvio-padrão & 12,45 \\
\hline & Mínimo & 41,67 \\
\hline & Máximo & 83,33 \\
\hline \multirow[t]{5}{*}{ Meio-ambiente } & Média & 55,70 \\
\hline & Mediana & 56,25 \\
\hline & Desvio-padrão & 10,72 \\
\hline & Mínimo & 37,50 \\
\hline & Máximo & 81,25 \\
\hline \multirow[t]{5}{*}{ Geral } & Média & 61,95 \\
\hline & Mediana & 62,50 \\
\hline & Desvio-padrão & 13,83 \\
\hline & Mínimo & 25,00 \\
\hline & Máximo & 87,50 \\
\hline
\end{tabular}




\section{DISCUSSÃO}

A compreensão do conteúdo da QV na velhice é fundamental ao desenvolvimento de iniciativas visando a prevenção e reabilitação nos vários contextos da vida do indivíduo e a promover o bem-estar dos idosos. ${ }^{18}$

O presente estudo apresentou dados sociodemográficos e clínicos semelhantes a outros estudos realizados em programas de $\mathrm{AD},{ }^{19} \mathrm{e} \mathrm{em}$ especial realizados no PADI, ${ }^{20-22}$ já bastante discutidos na literatura.

Todos os pacientes relataram estar satisfeitos com o cuidador e com os cuidados recebidos em casa. É importante destacar que as entrevistas foram realizadas no domicílio dos pacientes, o que pode ter influenciado no padrão de respostas. Paskulin et al. ${ }^{19}$ relataram que as condições familiares são determinantes para satisfação do cuidado recebido; assim, a inter-relação da família e sua situação socioeconômica irão definir também a qualidade de cuidado. A visão dos acompanhantes sobre a QV tanto dos idosos saudáveis como aqueles com doença de Alzheimer que estão sob seus cuidados tende a ser pior do que dos próprios sujeitos, tornando a avaliação de QV pelos acompanhantes, até certo ponto útil, porém pouco confiável ${ }^{13}$, não tendo sido empregada neste estudo.

Em relação às vantagens $\mathrm{da} A D$, quando comparado ao atendimento recebido na internação hospitalar, destacam-se maior liberdade, tranquilidade, estar com a família, escolher os horários, não precisar ver o sofrimento dos outros e não precisar esperar sua vez para ser atendido, contribuindo para uma maior satisfação em relação aos aspectos específicos do dia a dia.

Os idosos mais jovens, ou seja, com menor idade (de 60 a 80 anos), apresentaram neste estudo impacto negativo na QV nos domínios psicológico e ambiental. Talvez isso se deva ao fato de que esses idosos mais jovens estariam mais fragilizados e debilitados do que os idosos mais velhos, ou que percebem maior decrepitude numa idade em que esse grau de declínio funcional não seria esperado e, portanto, não sendo algo atribuído como "próprio da idade". Esta condição de insatisfação provavelmente gera escores mais baixos na avaliação da QV. Revisão de literatura feita por Diener \& Suh em 1998 apud $d^{23}$ apontou como um dos fatores da relação entre eventos objetivos e subjetivos da QV na velhice o fato de que os jovens são mais pessimistas e exigentes quanto à QV do que os idosos, o que também pode explicar a pior pontuação da QV pelos idosos mais jovens.

O número de medicamentos associou-se aos domínios geral e psicológico da QV. Seu uso pela população idosa, em virtude da prevalência de comorbidades crônicas, reflete uma porcentagem significativa com média de uso de especialidades terapêuticas de quatro medicamentos por idoso ${ }^{24}$ e, geralmente, o uso desses medicamentos iniciase antes dos 60 anos, aumentando a possibilidade de uso com o avançar da idade. ${ }^{25}$ Para controlar a evolução das doenças crônicas e incapacidades, há a necessidade da intervenção médica, na maioria das vezes farmacológica, não para cura, mas melhorar a vida do indivíduo em outros aspectos, fornecendo suporte e encorajando-o para enfrentar as dificuldades impostas a sua vida. Porém, as restrições geralmente impostas por essas doenças e incapacidades limitam os idosos as atividades prazerosas e ao envolvimento social relacionando-se com a depressão e com afetos negativos. ${ }^{23}$

Algumas desordens crônicas são prevalentes na população idosa, repercutindo como um fator de impacto na QV. ${ }^{26}$ Neste estudo, verificou-se relação entre o número de comorbidades e os domínios físico e meio ambiente, e, entre as afecções, apenas as psiquiátricas apresentou associação com a avaliação da QV.

A presença de afecções psiquiátricas influencia negativamente a QV de modo significativo no domínio social do WHOQOL-Bref. A EDG diminuiu o escore dos domínios físico, psicológico, meio ambiente e geral, mostrando que os sintomas depressivos afetam negativamente a QV. Isso pode acontecer, segundo Pereira (2002 
apud ${ }^{27}$ ), devido às mudanças que ocorrem nas diferentes fases do envelhecimento, desde alterações físicas até a desvalorização social consequente $\mathrm{da}$ aposentadoria, considerando o sentimento e entendimento dos idosos diante dessas situações, seus ganhos e perdas psicológicas, suas frustrações e aspirações, que influenciam na avaliação subjetiva da QV. Resultados semelhantes foram encontrados no estudo de Scocco et al., ${ }^{13}$ onde piores escores de sintomas depressão, medidos pela EDG, correlacionaram-se a piores escores de QV medida pelo WHOQOL-100.

Russo et al., ${ }^{28} \mathrm{em}$ seu estudo sobre depressão e função física, mostraram que em idosos da comunidade, com idade entre 80 anos ou mais, a depressão influenciou negativamente $\mathrm{O}$ desempenho físico e as medidas do estado funcional. Tais complicações estão associadas a limitações em várias dimensões do bem-estar e da função social. ${ }^{29}$ Assim, a avaliação da QV na velhice tem sido associada a questões de independência e autonomia, e a dependência do idoso resulta das alterações biológicas (incapacidades) e de mudanças nas exigências sociais. ${ }^{30}$ Naumann et al..$^{31}$, em estudo sobre medida de QV por meio do WHOQOL-Brefem pacientes com depressão, observaram que o escore da QV estava fortemente correlacionado com a severidade da depressão, número de sintomas físicos auto-relatados e saúde geral; entretanto, não encontraram relação entre o diagnóstico da comorbidade e o escore de QV, como visto no presente trabalho, já que os casos com diagnóstico de depressão foram incluídos nas afecções psiquiátricas.

No presente estudo, é possível encontrar ainda idosos com deficiências cognitivas leves dentre aqueles que apresentavam afecções psiquiátricas, variável relacionada ao domínio relações sociais do WHOQOL-bref. Muangpaisan et al. ${ }^{32}$ empregaram o mesmo questionário de QV e observaram que os idosos tailandeses da comunidade, com deficiência cognitiva leve, apresentaram piores escores apenas no domínio psicológico de QV quando comparados a idosos sem essa afecção. Os piores escores na EDG dos idosos com a deficiência cognitiva leve se associaram aos piores escores nos domínios físico e psicológico do WHOQOL-Bref, assim como ser mais velho (70 e mais anos) correlacionou-se ao domínio físico e viver só, ao domínio relações sociais. $^{32}$

Dentre as doenças mais frequentemente encontradas, estão as afecções musculoesqueléticas. Notou-se, neste estudo, que os idosos com afecções musculoesqueléticas apresentaram prejuízo na QV em todos os domínios avaliados pelo $\mathrm{WHOQOL-Bref}$, provavelmente porque elas podem impedir ou limitar as atividades dos idosos, dificultando o sair de casa para consultas médicas ou atividades de lazer. Essa restrição leva o indivíduo a apresentar declínio funcional, aumento da dependência, perda da autonomia, isolamento social e depressão, o que leva prejuízos à funcionalidade física, psíquica e social, ${ }^{33}$ o que poderia explicar o prejuízo na QV em todos os domínios. Tal fato justifica a presença acentuada de idosos com afecções musculoesqueléticas em programa de $\mathrm{AD}$, uma vez que a gravidade clínica é a que mais influencia a QV nesse contexto, e não as condições sociais em que o idoso vive.

As queixas de dor (65,2\% dos idosos) e EVA, com média de 2,39 de dor, associaram-se ao domínio geral da QV. As queixas álgicas, acompanhadas ou não de incapacidades, podem ser um fator que contribui para uma pior QV, discutindo-se um paradoxo em relação a incapacidade e QV, em que indivíduos com comprometimento funcional podem referir boa ou excelente QV, refletindo um equilíbrio entre corpo, mente e espírito. ${ }^{26}$ No presente estudo, os idosos apresentaram-se mais fragilizados, possuindo diversas doenças crônicas que por sua vez causam dor e, consequentemente, atividades de lazer em ambiente externo podem tornar-se difíceis ou dolorosas.

Reis ${ }^{26}$ observou que as práticas das atividades de lazer, analisadas em função do ambiente e da companhia, sobressaem-se as atividades individuais, no ambiente doméstico do idoso. Xavier et al. ${ }^{34}$ verificaram que as atividades mencionadas como fonte de prazer estavam associadas à "ideia de produtividade" com uma 
finalidade no dia a dia. A presença de dor é umas das características do perfil dos idosos em $\mathrm{AD}$, o que justifica o porquê desses idosos relatarem visão pessimista sobre o estilo de vida.

Os idosos, em sua maioria $(65,2 \%)$, relatam ter a percepção subjetiva da visão excelente ou boa, conferindo-lhes segurança, uma vez que a acuidade visual diminuída se associa aos fatores de risco à dependência em realizar as atividades de vida diária e atividades instrumentais de vida diária. ${ }^{35}$

O prejuízo funcional também tem repercussão negativa na QV. ${ }^{26} \mathrm{~A}$ capacidade funcional mostrou associação com os domínios físico, psicológico e relações sociais da QV. Geralmente dimensionada em termos da habilidade e independência para realizar determinadas atividades, é um dos grandes componentes da saúde no idoso e, recentemente, aponta como um componente importante para a avaliação da saúde dessa população. ${ }^{36} \mathrm{Geib},{ }^{37} \mathrm{em}$ seu estudo sobre associação entre capacidade funcional e QV de idosos da comunidade que moram sozinhos no município de São Paulo, observou que a maioria dos idosos afirmou não querer morar com os familiares, preferindo manter-se em sua própria residência até quando puderem fazer essa escolha, sendo que foi observado também que esses idosos demonstraram, em média, ter boa QV. A mesma autora $^{37}$ também verificou que a perda $\mathrm{da}$ capacidade funcional dessas pessoas não os impedia de viver sozinhos e independentes em suas residências, tendo em vista que o bem-estar do idoso, como ressaltado por Pereira et al., ${ }^{27}$ pode ser atingido independentemente da presença ou ausência de doenças, como resultado do equilíbrio entre as diversas dimensões da capacidade funcional.

O reduzido número amostral, que limita as associações entre as variáveis do presente estudo, pode ser justificado pela exclusão de vários pacientes do PADI, com presença de demência de moderada a grave, já que limitaria ou impediria a aplicação do $W H O Q O L-B r e f$.

Surpreendentemente, não houve correlações com dados estritamente sociais. Uma das razões para um idoso poder ser admitido no PADI é a condição social, clínica e funcional, ou seja, sujeitos que não têm condições de se locomover por falta de recursos ou devido sua condição clínica. ${ }^{22}$ A gravidade clínica é o que mais influencia a QV nesse contexto, e não as condições sociais em que o idoso vive, ao contrário do que foi encontrado por Muangpaisan et al., ${ }^{32}$ cujos sujeitos idosos com deficiência cognitiva leve percebiam pior QV no domínio psicológico conforme o estado financeiro fosse autoavaliado como ruim ou regular.

Lopes et al. ${ }^{21}$ descreveram que o reconhecimento dos fatores que pioram o declínio funcional de idosos em AD auxilia os profissionais intervir de forma preventiva na saúde dessa população, focando a melhoria da QV. Da mesma maneira, reconhecer os fatores que implicam piora da QV desses idosos faz com que a equipe multidisciplinar aborde de forma mais específica a avaliação, o acompanhamento e o cuidado com esses indivíduos. Segundo Paschoal, ${ }^{33}$ uma avaliação geriátrica elaborada com enfoque nas alterações do envelhecimento e nos fatores que influenciam esse processo, com abordagem mais específica para essa população, é capaz de controlar a evolução das doenças crônicodegenerativas, minimizando o risco de incapacidades, com intuito de aumentar a expectativa e a QV.

Os múltiplos aspectos que caracterizam o processo de envelhecimento preconizam atenção abrangente à saúde do idoso, de maneira multidimensional e multiprofissional, conforme almeja a OMS, em que visa não somente ao controle das doenças, mas sobretudo ao bem-estar físico, psíquico e social, ou seja, a melhora da QV dos idosos. ${ }^{38} \mathrm{~A}$ atenção interdisciplinar e domiciliária ao idoso fragilizado é de extrema importância para a manutenção e melhora da sua QV.

Este é o primeiro estudo a analisar os fatores que se associam à QV de idosos que estão em atendimento domiciliário no Programa de Assistência Domiciliária ao Idoso (PADI) da disciplina de Geriatria e Gerontologia da Unifesp 
de orientação estritamente gerontológica. Algumas limitações, entretanto, merecem ser ponderadas: (1) a amostra contou com um número reduzido de sujeitos que já estavam sendo beneficiados pelas ações de saúde da modalidade de assistência, cada qual por um período de tempo diferente, o que poderia influenciar as percepções dos sujeitos em função de graus de satisfação diferentes com o programa; (2) o PADI alicerçou-se numa visão de $A D$ característica, marcada pela multiprofissionalidade e por orientações aos cuidadores e não por intervenções contínuas de reabilitação, diferentemente de outras propostas possíveis de AD gerontológica.

Deste modo, recomenda-se cautela na generalização dos resultados para toda a população idosa atendida por esse tipo de assistência à saúde. Como as necessidades individuais de cuidados e as possibilidades de atuação intervencionista são diversas, a $\mathrm{AD}$ deve ser concebida conceitualmente como um "guarda-chuva" sempre acompanhado dos subcomponentes que descrevem o nível de atenção à saúde que é oferecido, bem como seus objetivos subjacentes. $^{6}$

Estudos futuros acerca do impacto de intervenções de saúde sobre a QV do idoso

\section{REFERÊNCIAS}

1. Ramos LR. Fatores determinantes do envelhecimento saudável em idosos residentes em centro urbano: projeto Epidoso. Cad Saúd Pública 2003; 19(3): 793-798.

2. Ferreira ABH. Novo Aurélio: o dicionário da língua portuguesa. Rio de Janeiro: Nova Fronteira;1999.p. 1099.

3. Carletti SMM, Rejani MI. Atenção domiciliária ao paciente idoso. In: Netto MP. Gerontologia: a velhice e o envelhecimento em visão globalizada. São Paulo: Atheneu; 2002. p. 3-17.

4. Gordilho A, et al. Desafios enfrentados no terceiro milênio pelo setor saúde na atenção integral ao idoso. Análise de dados 2001; 9(4): 138-153.

5. Sayeg MA. Envelhecimento bem sucedido e o auto-cuidado: algumas reflexões. Arq Geriatr Geront 1998; 2(3): 96-98. atendido em domicílio são necessários para se verificar a validade desse parâmetro como medida de resultados das ações planejadas sob o olhar interdisciplinar e de caráter multiprofissional.

\section{CONCLUSÕES}

No presente estudo, percebemos a influência negativa sobre a QV de idosos em AD: ser mais jovem (domínios psicológico e ambiente), queixa de dor (geral), maior intensidade de dor (geral), polifarmacoterapia (geral e psicológico), maior número de comorbidades (físico e ambiente), presença de sintomas depressivos (geral, físico, psicológico e ambiente), comprometimento funcional (físico, psicológico e relações sociais) e presença de afecções psiquiátricas (social).

Esses resultados preliminares fornecem indícios dos aspectos mais relevantes a se considerar em abordagens intervencionistas no contexto domiciliar. Realizadas por uma equipe multiprofissional e interdisciplinar, as intervenções multifatoriais seriam o ponto-chave do planejamento assistencial nessa modalidade que prevê a QV como uma das principais medidas de eficácia.
6. Thome B, et al. Home care with regard to definition, care recipients, content and outcome: systematic literature review. J. Clin Nurs 2003 nov; 12(6): 860-872.

7. Zahar SEV, et al. Avaliação de qualidade de vida na menopausa. Reprod Clim 2001; 16(3): 163-166.

8. Fleck MPA, et al. Aplicação da versão em português do instrumento de avaliação de qualidade de vida da Organização Mundial da Saúde (WHOQOL-100). Rev Saude Publica 1999;33(2):198-205.

9. Chachamovich E, et al. Assessment of the psychometric a sample of brazilian older adults. Int Psychogeriatr 2007 aug; 19(4): 635-46.

10. Bertolucci PHF, et al. The mini-mental state examination in a general population: impact of educational status. Arq Neuropsiquiatr 1994; 52(1): 1-7. 
11. Trigg R, et al. Can people with mild to moderate dementia provide reliable answers about their quality of life? Age Ageing 2007 nov; 36(6): 663669.

12. Longsdon RG, et al. Assessing quality of life in older adults with cognitive impairment. Psych Med 2002; 64: 510-519.

13. Scocco P, et al. Role of depressive and cognitive status in self-reported evaluation of quality of life in older people: comparing proxy and physician perspectives. Age Ageing 2006 mar; 35(2): 166-171.

14. Campbell WI, Lewis S. Visual analogue measurement of pain. Ulster Medical Journal 1990;59(2): 149-154.

15. Ramos LR, et al. Significance e management of disability among urban elderly residents in Brazil. J. Cross Cult Gerontol 1993; 8(8): 313-323.

16. Sheik JI, Yesavage JA. Geriatric depression scale: recent evidence and development of a shorter version. Clin Gerontol 1986; 5: 165-172.

17. Organização Mundial de saúde (Brasil). Versão em português dos instrumentos de avaliação de qualidade de vida (WHOQOL): projeto desenvolvido no Brasil pelo grupo de estudos em qualidade de vida. Universidade Federal do Rio Grande do Sul. Disponível em: http:// www.ufrgs.br/psiq/whoqol.html\#sumario

18. Neri AL. Qualidade de vida na velhice. In: Rebelatto JR, Morelli JGS. A prática assistência ao idoso. São Paulo: Manole; 2004. p. 4-29.

19. Paskulin LMG, Dias VRFG. Como é ser cuidado em casa: as percepções dos clientes. Rev Bras Enfermagem 2002; 55(2): 140-145.

20. Ricci NA, Kubota MT, Cordeiro RC. Concordância de observações sobre a capacidade functional de idosos em assistência domiciliar. Rev Saúde Pública 2005; 39(4): 655662.

21. Lopes AB, Gazzola JM, Lemos ND, Ricci NA. Independência funcional e os fatores que a influenciam no âmbito de assistência domiciliária ao idoso. Rev. Bras. Geriatr Gerontol 2007; 10(3): 285-300.

22. Bastos CC, Lemos ND, Mello AN. Perfil clínico-demográfico dos pacientes inseridos em um programa de assistência domiciliária no município de São Paulo. Revista Kairós 2007; 10(2): 205-224.

23. Paschoal SMP. Qualidade de vida na velhice. In: Freitas EV, et al. Tratado de geriatria e gerontologia. 2. ed. Rio de Janeiro: Guanabara Koogan; 2006. p.147-153.
24. Mosegui GBG, et al. Avaliação da qualidade do uso de medicamentos em idosos. Rev Saúde Pública 1999; 33(5): 437-444.

25. Bardel A, Wallander M, Svardsudd K. Report current use of prescription drugs an some of its determinants among 35 to 65 years-old women in Mid-Sweden: a population-based study. J. Clin Epidemiol 2000; 53(6): 637-643.

26. Reis ALB. Caracterização da qualidade de vida de idosos admitidos em serviço de reabilitação gerontológica. São Paulo. Dissertação [Mestrado em Reabilitação]-Universidade Federal de São Paulo; 2004.

27. Pereira RJ, et al. Contribuição dos domínios físico, social, psicológico e ambiental para a qualidade de vida global de idosos. Rev Psiquiatr Rio Gd. Sul 2006; 28(1): 27-38.

28. Russo A, et al. Depression and physical function: results from the aging and longevity study in the sirente geographic area (SIRENTE Study). J Geriatr Psychiatry Neurol 2007; 20(3): 131-137.

29. Fleck MPA, et al. Associação entre sintomas depressivos e funcionamento social em cuidados primários à saúde. Rev Saúde Pública 2002; 36(4): 431-438.

30. Sousa L, Galante H, Figueiredo D. Qualidade de vida e bem-estar dos idosos: um estudo exploratório na população portuguesa. Rev Saude Publica 2003; 37(3): 364-371.

31. Naumann VJ, Byrne GJA. WHOQOL- BREF as a measure of quality of life in older patients with depression. International Psychogeriatrics 2004; 16(2): 159-173.

32. Muangpaisan W, et al. Quality of life of the community-based patients with mild cognitive impairment. Geriatr Gerontol Int 2008; 8: 80-85.

33. Paschoal SMP. Qualidade de vida do idoso: elaboração de um instrumento que privilegia sua opinião . São Paulo.Dissertação [ Mestrado em Medicina] - Universidade de São Paulo; 2000.

34. Xavier FMF, et al. A definição dos idosos de qualidade de vida. Rev Bras Psiquiatr 2003; 25(1): 40-51.

35. Nakatani AYK, et al. Perfil sócio-demográfico e avaliação funcional de idosos atendidos por uma equipe de saúde da família na periferia de Goiânia, Goiás. Revista Soc Bras Clin Med 2003; 1(5): 131-136.

36. Cornoni-Huntley J, et al. Established populations for epidemiologic studies of the elderly. Washington: Department of health an human services; 1986. 
37. Geib S. Associação entre capacidade funcional e qualidade de vida de idosos da comunidade que moram sozinhos no município de São Paulo.São Paulo Dissertação [Mestrado em Medicina]Universidade Federal de São Paulo ; 2001.
38. Papaléo Netto M. O estudo da velhice no século $\mathrm{XX}$ : histórico, definição do campo e termos básicos. In: Freitas EV, et al. Tratado de geriatria e gerontologia. Rio de Janeiro: GuanabaraKoogan; 2002. p. 2-10.

Recebido: $27 / 11 / 2008$

Revisado: 22/9/2009

Aprovado: 12/1/2010 